\title{
An adjustable approach to multi-criteria group decision-making based on a preference relationship under fuzzy soft information
}

\begin{abstract}
Group decision-making is a collaborative process to find optimal alternative based on an aggregation judgment. Various techniques have been suggested to solve decision-making problems; however, the rapid growth of uncertainties in industry and organizations highlights the application of fuzzy set theory and soft set theory in this area. In this regard, fuzzy soft model can be considered as an efficient tool in decision-making. To date, different algorithms have been proposed for solving collective decision-making problems based on fuzzy soft set theory. In order to reach the process of consensus, the existing methods have mostly used the t-norms, such as "AND" operator which can be successfully applied to individual decisionmaking problems including multi-source datasets. However, such approaches fail to consider multi-observer problems in group decision-making processes. Additionally, in the selection step, the existing methods lack a comprehensive priority approach; they focus on a hierarchical preference which ignores incomparable alternatives. To overcome these issues, this paper proposes an adjustable multi-criteria group decision-making approach based on a preference relationship of fuzzy soft sets. First, we construct two topological spaces over the set of objects, and then, develop a preference relationship of objects by using open sets of these two topologies. A multi-phase method is then designed to rank objects in multi-criteria group decision-making problems based on such preference relationship. We also extend the proposed algorithm to weighted case in order to have a higher level of adaptability with realworld problems. Dataset from "www.booking.com" Web site is applied to show the capability of this new method in comparison with results from the well-known literature approaches.
\end{abstract}

Keyword: Fuzzy soft set; Weighted fuzzy soft set; Preference relationship; Multi-criteria group decision-making 